\title{
Self interactions of Supernova neutrinos
}

\author{
Antonio Marrone* \\ Dipartimento di Fisica and Sezione INFN di Bari, Via Amendola 173, 70126 Bari, Italy \\ E-mail: antonio.marrone@ba.infn.it
}

\section{Gianluigi Fogli}

Dipartimento di Fisica and Sezione INFN di Bari, Via Amendola 173, 70126 Bari, Italy

\section{Eligio Lisi}

Istituto Nazionale di Fisica Nucleare, Sezione di Bari, Via Orabona 4, 70126 Bari, Italy

\section{Irene Tamborra}

Dipartimento di Fisica and Sezione INFN di Bari, Via Amendola 173, 70126 Bari, Italy

\begin{abstract}
We studied the effect on the supernova neutrino spectra of collective flavor transitions induced by self-interactions. Collective oscillations produce splits and swaps of neutrino and antineutrino spectra. In particular, we considered the case of one spectral split for neutrinos and one for antineutrinos, and we determined an analytic condition to predict the split energies.
\end{abstract}

35th International Conference of High Energy Physics - ICHEP2010,

July 22-28, 2010

Paris France

*Speaker. 

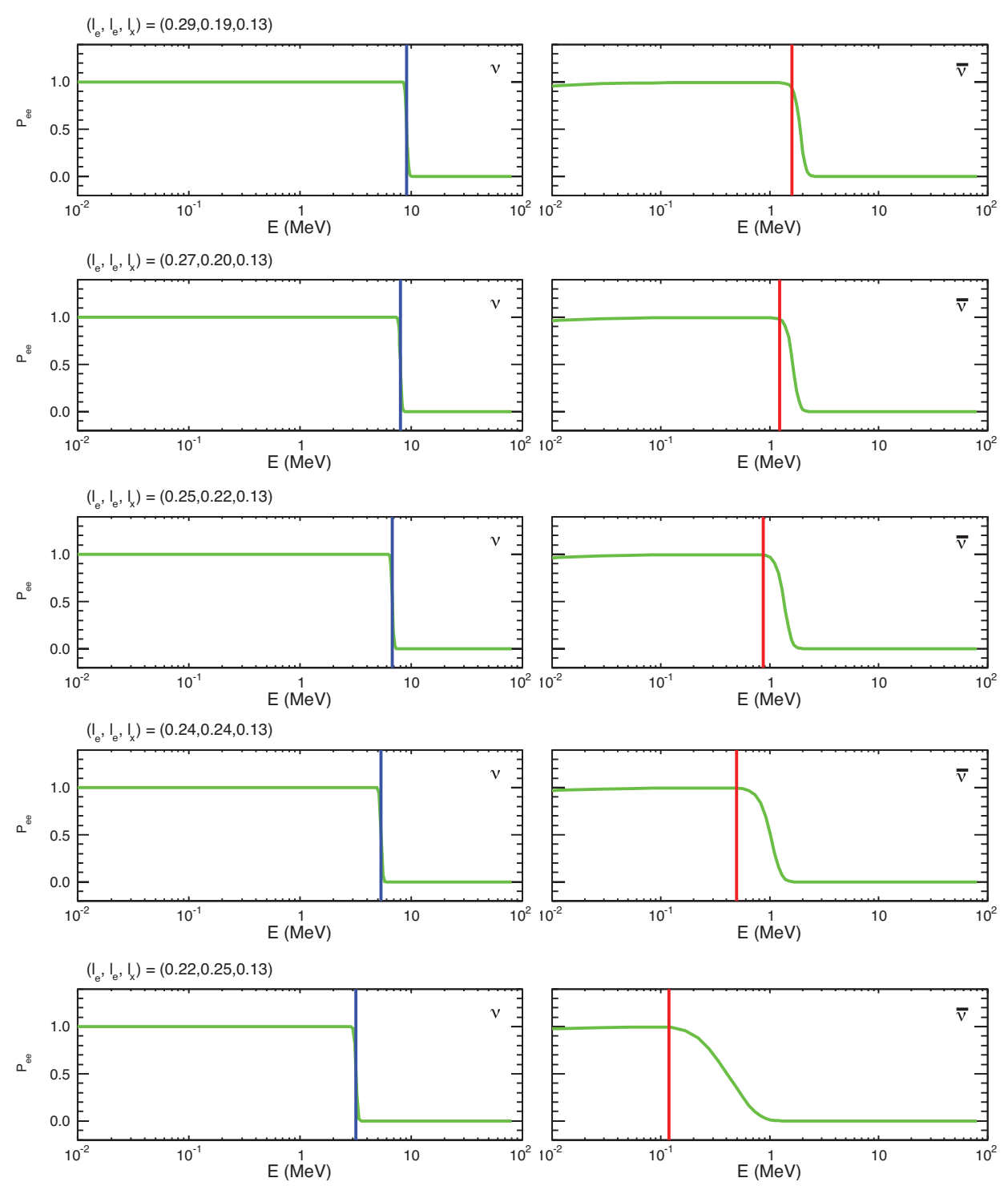

Figure 1: $v_{e}$ and $\bar{v}_{e}$ survival probability $\left(D_{z}>0\right)$.

\section{Introduction}

Supernova neutrino oscillations are a very useful tool to study neutrino properties [1]. When the neutrinos leave the neutrinosphere, they undergo ordinary vacuum and matter oscillations, but if the neutrino density is very high, they can also feel their own potential and they can oscillate in a collective way. Collective oscillations can be very different from ordinary oscillations. Selfinteraction effects are important at a distance of few tens of kilometers from the neutrinosphere, where the self-interaction potential $\mu$ is of the same order of the matter potential $\lambda$ or of the vacuum neutrino frequency $\omega$.

In this work we considered two families of neutrinos $\left(v_{e}, v_{x}\right)$, and we used the single angle approximation, without matter effects. The supernova total luminosity in neutrinos was fixed, but 

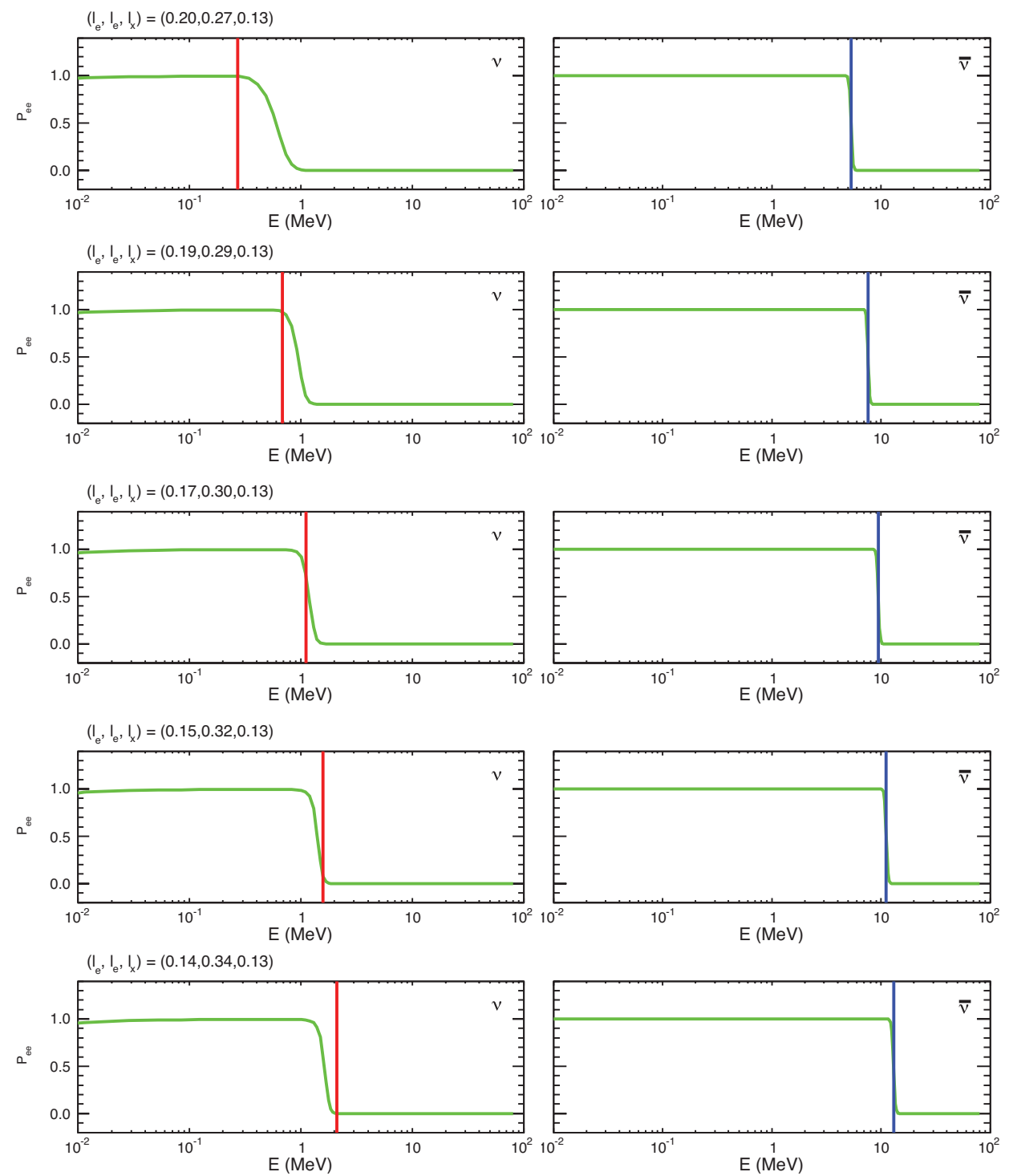

Figure 2: $v_{e}$ and $\bar{v}_{e}$ survival probability $\left(D_{z}<0\right)$

the relative fractional luminosity $l_{\alpha}$ in the flavor $\alpha$ was allowed to vary. We imposed the constraint $l_{e}+l_{\bar{e}}+4 l_{x}=1$ [2] (the factor 4 in front of $l_{x}$ accounts for the two flavor $v_{\mu}$ and $v_{\tau}$ and the relative antineutrinos).

\section{Split Energies}

In general, collective transitions can produce single or multiple spectral splits, both for neutrinos and antineutrinos. We investigated the case in which there is only one split for neutrinos and only one split for antineutrinos. Some of the features of the collective oscillations can be understood by means of three global vectors, $\mathbf{J}, \overline{\mathbf{J}}$ and $\mathbf{D}=\mathbf{J}-\overline{\mathbf{J}}$ [3], constructed by opportunely adding the polarization vectors $\mathbf{P}(E)$ and $\overline{\mathbf{P}}(E)$, that describe the oscillations of each $v$ and $\bar{v}$ energy mode 
$E$, in the density matrix formalism. In our simulations, we observed that a single split in both the neutrino and the antineutrino sector occurs when the global vectors projections $J_{z}$ and $\bar{J}_{z}$ are initially both positive. We also observed that one of the two split energies is of order of $\sim 1 \mathrm{MeV}$ (LE) and it is less sharp than the other, that is of order of $\sim 10 \mathrm{MeV}$ (HE). Moreover, we noted that the low energy split occurs for antineutrinos when $D_{z}>0$ and for neutrinos when $D_{z}<0$.

In a first approximation, we can simply disregard the LE split: in this case the HE split can be obtained from an integral equation expressing lepton number conservation [3]. The equation of motion of the polarization vector (see [3]) can have a resonance-like behavior in the (anti)neutrino sector if $D_{z}<0\left(D_{z}>0\right)$. The resonance happens when the vacuum frequency of a mode is of the order of the self-interaction potential times $D_{z}$, i.e. when $\omega \simeq \mu D_{z}$. After encountering the resonance, the corresponding mode proceeds with its own vacuum frequency. Note that the previous equation has two unknowns, the energy of the mode and the radius at which the resonance occurs. When the collective oscillations begin (bipolar regime), all the mode precess with the same frequency $\omega_{\text {bipolar }} \simeq \sqrt{\mu \omega_{\text {ave }} Q_{z}} \sim \sqrt{\mu \omega_{\text {ave }} D_{z}}$ (see [4] for a definition of $Q_{z}$ and [3] for a definition of $\omega_{\text {ave }}$ ). When $\omega \gtrsim \omega_{\text {bipolar }}$, even after the start of the collective oscillations, the corresponding mode remains decoupled, while for $\omega<\omega_{\text {bipolar }}$ the mode participate to the collective oscillations. The condition $\omega=\omega_{\text {bipolar }}$, at the onset of bipolar oscillations, gives the second equation that allows us to calculate the lower split energy. Figure 1 shows the predictions fo the HE split (blue vertical line) and the LE split (red line), together with the calculated survival $v_{e}$ probability, in the case $D_{z}>0$ and for different choices of the fractional luminosities. Figure 2 is analogous to Figure 1 but for the case $D_{z}<0$, in which the secondary low energy split is in the antineutrino sector.

\section{Conclusions}

We investigated supernova neutrino oscillations in two generations, in the single angle approximation, in the absence of the matter potential, and allowing the fractional luminosities in each neutrino flavor to vary. We considered only cases in which there is one split in the neutrino and in the antineutrino spectrum. We found an analytic condition to predict the split energies, by solving two equations. The first equation expresses the lepton conservation number and the second one is the condition of equality between the vacuum frequency of a mode and the frequency of the bipolar oscillations. The predicted split energies are in good agreement with the results of the numerical simulations.

\section{References}

[1] G. G. Raffelt, "Supernova neutrino observations: What can we learn?”, astro-ph/0701677.

[2] G. L. Fogli, E. Lisi, A. Marrone and I. Tamborra, "Supernova neutrinos and antineutrinos: Ternary luminosity diagram and spectral split patterns," JCAP 0910:002,2009.

[3] G. L. Fogli, E. Lisi, A. Marrone and A. Mirizzi, "Collective neutrino flavor transitions in supernovae and the role of trajectory averaging," JCAP 0712:010 (2007). arXiv:0707.1998 [hep-ph].

[4] S. Hannestad, G. G. Raffelt, G. Sigl and Y. Y. Y. Wong, "Self-induced conversion in dense neutrino gases: Pendulum in flavour space,” Phys. Rev. D 74, 105010 (2006) [astro-ph/0608695]. 